\section{(2) OPEN ACCESS}

\title{
Gut dysbiosis induces the development of pre- eclampsia through bacterial translocation
}

\author{
Xia Chen (1) , ${ }^{1}$ Pan Li, ${ }^{2}$ Mian Liu, ${ }^{1}$ Huimin Zheng, ${ }^{2,3}$ Yan He, ${ }^{2}$ Mu-Xuan Chen, ${ }^{2}$ \\ Wenli Tang, ${ }^{2}$ Xiaojing Yue, ${ }^{1}$ Yongxin Huang, ${ }^{1}$ Lingling Zhuang, ${ }^{4}$ Zhijian Wang, \\ Mei Zhong, ${ }^{1}$ Guibao Ke, ${ }^{5}$ Haoyue Hu, ${ }^{1}$ Yinglin Feng, ${ }^{1}$ Yun Chen, ${ }^{1}$ Yanhong Yu, \\ Hongwei Zhou, ${ }^{2}$ Liping Huang'
}

Additional material is published online only. To view, please visit the journal online (http://dx.doi.org/10.1136/ gutjnl-2019-319101).

For numbered affiliations see end of article.

\section{Correspondence to}

Professor Liping Huang, Department of Obstetrics and Gynecology, Southern Medical University Nanfang Hospital, Guangzhou, Guangdong, China; Iphuang2006@126.com, Professor Hongwei Zhou, Microbiome Medicine Center, Division of Laboratory Medicine, Zhujiang Hospital, Southern Medical University, Guangzhou, Guangdong, China; hzhou@smu.edu.cn and Professor Yanhong Yu, Department of Obstetrics and Gynecology, Southern Medical University Nanfang Hospital, Guangzhou, Guangdong, China; yuyh1010@hotmail.com

$\mathrm{XC}, \mathrm{PL}$ and $\mathrm{ML}$ contributed equally.

Received 15 May 2019 Revised 11 December 2019 Accepted 14 December 2019

Published Online First 3 January 2020

\begin{abstract}
Objective Pre-eclampsia (PE) is one of the malignant metabolic diseases that complicate pregnancy. Gut dysbiosis has been identified for causing metabolic diseases, but the role of gut microbiome in the pathogenesis of PE remains unknown.
\end{abstract}

Design We performed a case-control study to compare the faecal microbiome of PE and normotensive pregnant women by $16 \mathrm{~S}$ ribosomal RNA (rRNA) sequencing. To address the causative relationship between gut dysbiosis and $P E$, we used faecal microbiota transplantation (FMT) in an antibiotic-treated mouse model. Finally, we determined the microbiome translocation and immune responses in human and mouse placental samples by 16S rRNA sequencing, quantitative PCR and in situ hybridisation.

Results Patients with PE showed reduced bacterial diversity with obvious dysbiosis. Opportunistic pathogens, particularly Fusobacterium and Veillonella, were enriched, whereas beneficial bacteria, including Faecalibacterium and Akkermansia, were markedly depleted in the PE group. The abundances of these discriminative bacteria were correlated with blood pressure (BP), proteinuria, aminotransferase and creatinine levels. On successful colonisation, the gut microbiome from patients with PE triggered a dramatic, increased pregestational BP of recipient mice, which further increased after gestation. In addition, the PE-transplanted group showed increased proteinuria, embryonic resorption and lower fetal and placental weights. Their T regulatory/helper-17 balance in the small intestine and spleen was disturbed with more severe intestinal leakage. In the placenta of both patients with PE and PE-FMT mice, the total bacteria, Fusobacterium, and inflammatory cytokine levels were significantly increased.

Conclusions This study suggests that the gut microbiome of patients with PE is dysbiotic and contributes to disease pathogenesis.

\section{INTRODUCTION}

(c) Author(s) (or their employer(s)) 2020. Re-use permitted under CC BY-NC. No commercial re-use. See rights and permissions. Published by BMJ.

To cite: Chen $X$, Li P, Liu M, et al. Gut 2020;69:513-522.
Pre-eclampsia (PE) is a disorder of pregnancy associated with new-onset hypertension, which occurs most often after 20 weeks of gestation combined with proteinuria. ${ }^{1} \mathrm{PE}$ affects $2 \%-8 \%$ of pregnancies and is associated with an increased risk of adverse pregnancy outcomes, including placental abruption, prematurity and intrauterine growth restriction. ${ }^{23}$

\section{Significance of this study}

What is already known on this subject?

- Pre-eclampsia (PE) is one of the leading causes of maternal and perinatal morbidity and mortality and lacks reliable prediction methods and detailed pathogenesis knowledge.

- PE can be considered as a type of metabolic disease that complicates pregnancy.

- Gut microbiota dysbiosis plays a causative role in the development of metabolic syndrome and complicated pregnancies.

What are the new findings?

- Inoculation of the gut microbiome from patients with PE triggered a significantly higher blood pressure before pregnancy and PE-like phenotypes during pregnancy, including worsened hypertension, exacerbation of proteinuria and intrauterine growth restriction.

- Higher levels of total bacteria and Fusobacterium and an altered microbiota profile were identified in PE placenta samples compared with in normotensive samples.

- The dysbiotic gut microbiome induced immune imbalances related to T-lymphocytes and intestinal barrier dysfunction, facilitating translocation of bacteria to the intrauterine cavity, ultimately eliciting inflammation in the placenta and contributing to poor placentation.

How might it impact on clinical practice in the foreseeable future?

- Gut microbiota screening shows potential for predicting PE onset and provides a new approach for the prevention and treatment of this disease.

To date, no first-trimester or second-trimester tests can reliably predict the development of all cases of $\mathrm{PE},{ }^{3}$ and thus, it is of particular interest to find effective and accessible clinical biomarkers in the field of obstetrics. The pathogenesis of this morbidity is still vague to date. ${ }^{2}$ Emerging studies have indicated that PE should be considered as a type of metabolic disease closely associated with metabolic disorders such as obesity, diabetes mellitus, insulin resistance, atherogenic dyslipidaemia and hyperglycaemia. ${ }^{4-6}$ 
The presence of glucose intolerance and dyslipidaemia, along with hypertension, can contribute to endothelial dysfunction, poor placentation and abnormal placental development, which are common pathological abnormalities in PE. ${ }^{7}$

Numerous studies have suggested that gut dysbiosis plays a causative role in the development of metabolic syndrome, chronic inflammation diseases and complicated pregnancies. ${ }^{89}$ Gut microbiota can facilitate insulin resistance by inducing the chronic inflammation in the host and causing fat accumulation by regulating genes related to energy metabolism, which plays an important role in the development of core symptoms in metabolic disorders. ${ }^{10}$ Gut microbiome dysbiosis is a key factor in blood pressure (BP) regulation ${ }^{11} 12$ and can contribute to the development of proteinuria and subsequently lead to kidney disease. $^{13}$

The composition of the gut microbiota in patients with PE and the relationship between the gut microbiota and PE have not been clarified so far. Recent studies suggested a disrupted gut microbiota composition in patients with $\mathrm{PE}$ in late pregnancy, ${ }^{14} 15$ but there is lack of causative analysis. In this study, we performed 16S ribosomal RNA (rRNA) gene sequencing on faecal samples to investigate the detailed profile of the gut microbiome and to determine the crucial role of the disordered gut microbiome in triggering PE-like phenotypes by faecal microbiota transplantation (FMT).

\section{MATERIALS AND METHODS Study subjects}

Women with PE and normotensive pregnant (NP) women without previous treatments were recruited in the third trimester from March 2017 to March 2018 in the Department of Obstetrics of the Nanfang Hospital, Southern Medical University, China. According to the current American College of Obstetricians and Gynaecologists criteria, ${ }^{2} \mathrm{PE}$ was defined as hypertension (systolic blood pressure (SBP) or diastolic blood pressure (DBP) of $\geq 140$ or $\geq 90 \mathrm{~mm} \mathrm{Hg}$ on two occasions at least 4 hours apart) and significant proteinuria ( $\geq 300 \mathrm{mg}$ protein per 24 hours' urine collection or protein/creatinine ratio of $\geq 0.3 \mathrm{mg} / \mathrm{dL}$ or dipstick reading of $2+$ in a random urine sample) after 20 weeks of gestation in a woman with a previously normal BP. In the absence of proteinuria, PE was defined when hypertension was associated with any features of end organ damage. PE with severe features was considered when either severe hypertension (SBP or DBP $\geq 160$ or $\geq 110 \mathrm{~mm} \mathrm{Hg}$ ), thrombocytopenia, impaired liver function, renal insufficiency pulmonary oedema, headache or visual disturbance was present. The clinical characteristics of the faecal and placenta donors are summarised in online supplementary tables S1 and S2, respectively.

\section{Animals and experimental protocol}

C57BL/6 mice were housed with a 12 hours' light-dark cycle in specific pathogen-free facilities. FMT was performed according to a modified method described previously. ${ }^{16}$ Briefly, female mice aged 6-8 weeks were administered antibiotics (vancomycin, $100 \mathrm{mg} / \mathrm{kg}$; neomycin sulfate, metronidazole and ampicillin, $200 \mathrm{mg} / \mathrm{kg}$ ) intragastrically once daily for five consecutive days. We randomly selected three patients with PE and three NP controls as faecal donors for FMT (online supplementary table S3). The faecal supernatants of three PE and three NP donors were mixed and used as a single source for the PE-FMT and NP-FMT mice, respectively. Following antibiotic treatment, the recipient mice were orally inoculated daily for three consecutive days and two times per week thereafter for 59 days with prepared faecal contents. Another group of female mice was gavaged as described previously, but the donor supernatant was replaced with phosphate-buffered saline (PBS). Six weeks after the first microbial administration, all female mice were housed with male mice at a 2-3:1 ratio overnight, and pregnancy was confirmed by the presence of vaginal spermatozoa. Mice harbouring microbiota from patients with PE and NP, as well as PBS, were referred to as PE-FMT, NP-FMT and control, respectively. The group and cage number of the mice involved in the study are shown in online supplementary table S4.

\section{Protein expression and biochemical analysis}

Protein was extracted from tissues with commercial lysis buffer. Western blotting was performed as described previously. ${ }^{17}$ Primary antibodies against zonula occluden (ZO)-1 (Invitrogen, Carlsbad, California, USA), ZO-2 (Abcam, Cambridge, UK), occludin (Invitrogen), claudin-4 (Abcam) and glyceraldehyde 3-phosphate dehydrogenase (GAPDH, Invitrogen) were used. Faecal albumin and urinary protein concentrations were determined with a mouse albumin ELISA kit (Bethyl Labs, Montgomery, Alabama, USA) according to the manufacturer's instructions. The endotoxin level was measured with a commercial kit (Bio-Swamp, Beijing, China).

\section{Gene expression analysis}

Total RNA was extracted with Trizol reagent (Invitrogen) according to the manufacturer's instructions. The reverse transcription reaction was carried out with reverse transcription enzyme (Toyobo, Shanghai, China). Quantitative real-time PCR was carried out on an ABI Q5 real-time PCR system and the specific primers for quantitative PCR are shown in online supplementary table S5.

\section{Flow cytometry}

Isolated immune cells were resuspended and stained with anti-CD4-FITC and/or anti-CD25-APC monoclonal antibodies (mAbs) for surface antigens and anti-Foxp3-PE or antiIL-17A-PE mAbs for intracellular antigens in accordance with the manufacturer's instructions (eBioscience, San Diego, California, USA). Fluorescence minus one controls were stained in parallel. Flow cytometry data acquisition was conducted using a BD LSRFortessa flow cytometer (BD Biosciences, San Jose, California, USA), and the analysis was performed by BD FACSDiva Software V.8.0.1 (BD Biosciences). The gating strategies are shown in online supplementary figure $\mathrm{S} 1$.

\section{In situ hybridisation}

In situ hybridisation (ISH) was performed following a modified protocol described by Choi et al. ${ }^{18} 19$ Briefly, paraffin-embedded sections were subjected to deparaffinisation, rehydration and permeabilisation. The probes used in this study were as follows: Eubacterial probes (EUB338 I-III) mixture complementary to a $16 \mathrm{~s}$ rRNA region specific for most bacteria; NON338, an oligonucleotide complementary to the probe EUB338, serving as a scrambled control for nonspecific binding; Fusobacteriumspecific probe, a genus-specific probe complementary to a $16 \mathrm{~S}$ rRNA region of Fusobacterium. All probes were 5'-labelled with digoxigenin, and antidigoxigenin/horseradish peroxidase antibodies were used as secondary antibodies. Tyramide signal amplification was performed to increase the detection sensitivity of bacterial signals. Six areas of each section were randomly photographed under a fluorescence microscope. The specific probes for ISH are shown in online supplementary table S6. 


\section{Bioinformatics processing}

Using QIIME V.1.9.1, we merged, applied quality control and clustered the 16S rRNA gene reads into operational taxonomic units (OTUs). Taxonomic groups were based on the Greengenes Database V.13_8 using closed reference to perform referenced-based OTU clustering. ${ }^{20}{ }^{21}$ Values for alpha diversity (Chao1 Index, Shannon's Index, Phylogenetic Diversity (PD) Whole Tree Index and observed OTUs), beta diversity (unweighted UniFrac distance metrics) and principal coordinate analysis (PCoA) employed based on the UniFrac metrics were generated by QIIME V.1.9.1. Permutational multivariate analysis of variance was performed to determine if the compositions of microbiota differed between groups. Linear discriminant analysis effect size (LEfSe) was performed to determine the features most likely to explain the differences between groups. ${ }^{22}$ To identify and filter the potential contaminant OTUs, decontam V.1.4. $0^{23}$ and SourceTracker V.1.0.1) ${ }^{24}$ were applied to the placenta samples and negative controls.

\section{Statistical analysis}

According to the different data, statistical analysis between groups were analysed using the Wilcoxon's rank-sum test, Student's $\mathrm{t}$-test or one-way analysis of variance. Correlation analyses were performed based on the Spearman's rho statistic. Rate comparisons were performed with Pearson's $\chi^{2}$ test or Fisher's exact test. Multiple hypotheses were adjusted using the Benjamini and Hochberg method. ${ }^{25}$ In the figures, $\mathrm{p}<0.05$ indicates statistical significance $(* \mathrm{p}<0.05, * * \mathrm{p}<0.01, * * \mathrm{p}<0.001)$. Statistical analyses and data visualisation were performed using R V.3.5.0 (under RStudio V.1.1.453), using the ggplot2 and corrplot packages.

Other materials and methods are described in the online supplementary materials and methods.

\section{RESULTS}

\section{Dysbiosis of gut microbiota in patients with PE}

A total of 85 normotensive women and 67 patients with PE were included in the present study. In the PE group, there were 40 patients with severe features (PESF) and 27 patients without severe features (PETSF). The PE group was further divided into an early onset of PE group (21 women) and a late onset of PE group (46 women) according to whether the disease was detected before 33 gestational weeks.

According to PCoA, the gut microbiome of the PE group differed significantly from that of the NP group using the unweighted UniFrac distance (figure 1A). Alpha-diversity indices, including Chao1 and observed OTU, were markedly decreased in the PE group (figure 1B,C and online supplementary figure S2A,B). We did not observe significant differences between the $\mathrm{PE}$ with and without severe features subgroups (online supplementary figure S3A-E) or between the early and late onset of PE subgroups (online supplementary figure S4A-E) for both alpha diversity and beta diversity.

The intestinal bacterial community consisted of 379 genera belonging to 18 phyla in patients with $\mathrm{PE}$ and in normotensive individuals. At both the phylum and genus levels, the microbial composition of patients with PE differed from that of the normotensive group (online supplementary figure S2C,D). To further identify which bacterial taxa were distinct between the PE and NP groups, we performed LEfSe analysis and identified seven genera showing significant differences (figure 1D). Moreover, the abundance comparisons of predominant genera showed that Clostridium, Dialister, Veillonella and Fusobacterium were significantly enriched, whereas Lachnospira, Akkermansia and Faecalibacterium were depleted in patients with PE (figure 1E-K). However, no significant differences of the abovementioned genera were observed among the PESF and PETSF subgroups (online supplementary figure S3F).

Phylogenetic Investigation of Communitiesby Reconstruction of Unobserved States (PICRUSt) analysis was performed to infer microbial metabolites and metabolic pathways. Pathways correlated with inflammation, including lipopolysaccharide biosynthesis and bacterial toxins, were enriched in patients with PE (online supplementary figure S2E,F). These key genera showed significant correlations with BP, proteinuria, impaired hepatic functions or other clinical manifestations based on Spearman correlation calculated for all participants (figure 1L). Notably, Veillonella and Fusobacterium were significantly correlated with most clinical parameters, including SBP, DBP, proteinuria, oedema levels, alanine aminotransferase, aspartate aminotransferase, serum creatinine and albumin. The correlation coefficients for Lachnospira, Akkermansia and Faecalibacterium were just opposite. However, no bacteria were significantly correlated with clinical parameters when testing only on the PE group, which could be due to reduced sample size. These results reveal profound changes in the intestinal microbiome structure of the PE group, indicating the importance of gut microbes in the development of PE.

\section{FMT induces the pre-eclamptic phenotype}

To further test whether alterations in the gut microbiota can cause the progression of PE in vivo, faecal microbiome from patients with PE were transplanted into mice. Following antibiotics treatment, the recipient mice were randomly divided into groups and orally inoculated with prepared faecal contents from donors with PE or NP (figure 2A). The gut microbial profiles of recipient mice were analysed by 16S RNA sequencing at 6 weeks post-transplantation. As expected, PCoA based on unweighted UniFrac distance matrices showed a significant effect in the FMT group on the mouse faecal microbiome (online supplementary figure S5A,B). From the donors, 71 of 128 genera successfully colonised the intestine of recipient mice (online supplementary figure $55 \mathrm{C}$ ). Additionally, 55\% of the shared genera were found to vary with the same trend across the microbiota of donors and recipient mice (online supplementary figure S5E). Moreover, at the genus level, the abundance variations in Coprococcus, Fusobacterium, Megamonas, Clostridium, Phascolarctobactarium and Akkermansia showed the same trend between mice and donors (online supplementary figure S5D,F). These results suggest that the faecal microbiota composition of human donors was successfully reproduced in recipient mice.

At 6 weeks post-transplantation, PE-FMT mice exhibited significantly higher pregestational SBP compared with NP-FMT and control mice. After pregnancy, the SBP of the PE-FMT group was further elevated from the fifth gestational day to the end of gestation (figure 2B and online supplementary figure S6A). In addition to worsening hypertension, the PE-FMT group showed a significantly higher urine protein concentration at 17 days of gestation, whereas urinary protein excretion was unchanged at 6 weeks post-FMT prior to mating (figure 2C). The number of live pups, fetal weight and placental weight were also decreased in the PE-FMT group, whereas the fetal resorption rate was increased compared with that in the NP-FMT and control groups (figure 2D,E). To determine how faecal bacteria from patients with PE affect the placenta, we examined the structure of the implantation sites. Histomorphological analysis revealed a significant structural change in the PE-FMT placenta, as the ratio between the two functional placental zones (labyrinth and junctional zones) was increased in placentas from the PE-FMT 
A

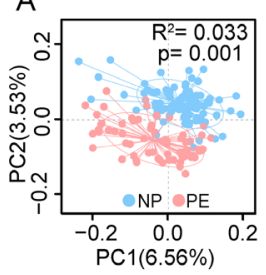

B

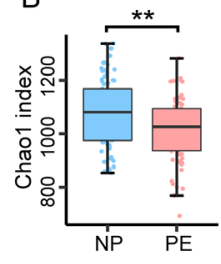

C

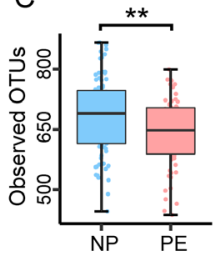

E

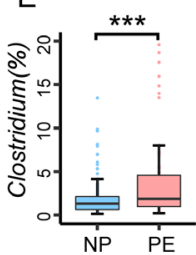

I
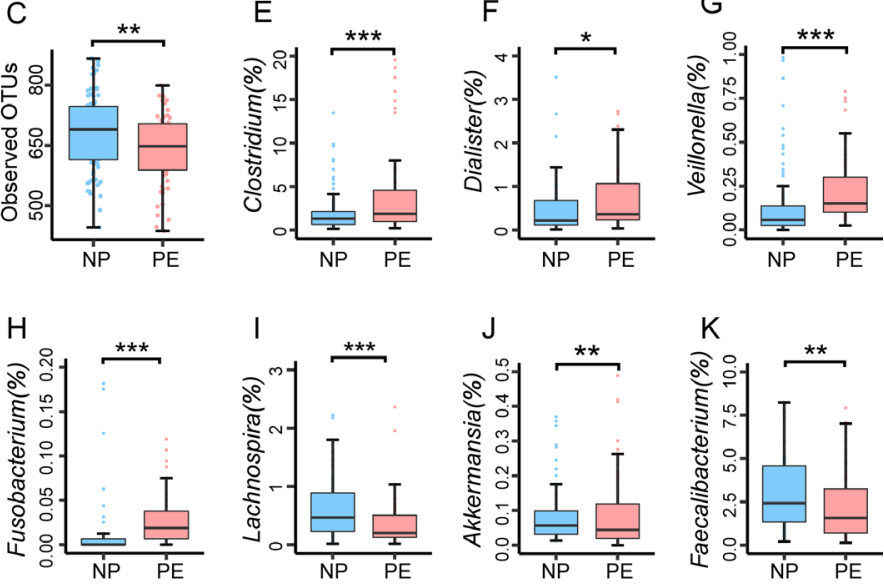

L

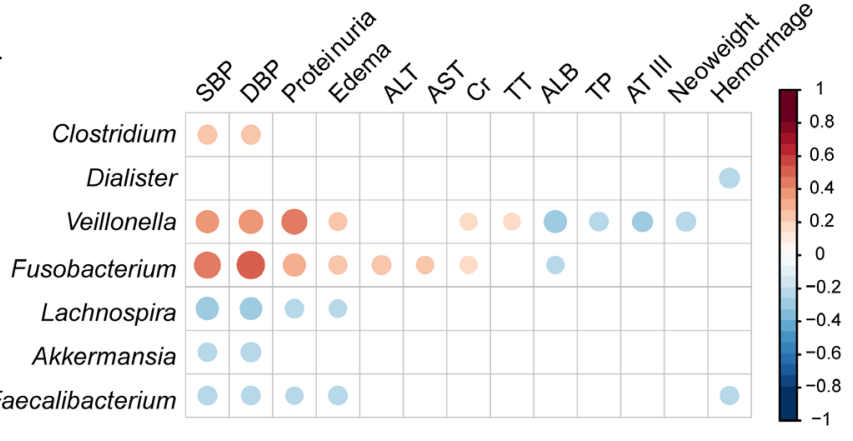

Figure 1 Dysbiosis of gut microbiota patterns in patients with PE and NP women. (A) Principal coordinate analysis based on unweighted UniFrac distances revealed that the NP bacterial communities clustered separately from PE bacterial communities, which were more similar to each other. Each circle represents a single sample, coloured by group. The eigenvalues of axe Principal coordinate (PC)1 and PC2 were 1.27 (6.56\%) and 0.73 $(3.53 \%)$, respectively. Difference in beta diversity between NP and PE were tested by permutational multivariate analysis of variance (Adonis). $(B, C)$ Comparison of alpha-diversity indices (Chao1 Index and observed operational taxonomic units) between PE and NP groups. ** indicates $\mathrm{p}<0.01$ by Wilcoxon rank-sum test. (D) linear discriminant analysis effect size identified the most differentially abundant taxa between the two groups. PE-enriched taxa are indicated with a positive LDA score, and taxa enriched in NP controls have a negative score. Only taxa meeting an LDA significant threshold of $>3$ are shown. (E-K) Relative abundances of Clostridium, Dialister, Veillonella, Fusobacterium, Lachnospira, Akkermansia and Faecalibacterium between PE and NP groups. * indicates $p<0.05$; * indicates $p<0.01$; and ${ }^{* * *}$ indicates $p<0.001$ by Wilcoxon rank-sum test. (L) Correlations between the relative abundance of selected bacterial genera and clinical parameters. Correlations with $p<0.05$ are shown. Spearman's rho statistic adjusted using the Benjamini and Hochberg method. AT-III, antithrombin III; ALB, albumin; ALT, alanine aminotransferase; AST, aspartate aminotransferase; $\mathrm{Cr}$, creatinine; DBP, diastolic blood pressure; LDA, linear discriminant analysis; NP, normotensive pregnant; $\mathrm{PE}$, pre-eclampsia; $\mathrm{SBP}$, systolic blood pressure; TP, total protein; TT, thrombin time.

group compared with those from the NP-FMT and control groups (figure $2 \mathrm{~F}$ ). In addition, differences in placental labyrinth morphology were evident in the PE-FMT group (figure 2G). The PE-FMT mice exhibited infarction and increased stromal collagen deposition in the labyrinth layer of the placenta. However, in the NP-FMT group, labyrinth morphology resembled that in controls. Furthermore, no visible histopathological abnormalities were observed in the kidney tissues (online supplementary figure S6B). These results show that PE-like phenotypes were induced by faecal bacterial transfer from patients with PE.

\section{FMT induces immunological imbalances}

Decreased regulatory $\mathrm{T}$ (Treg) cells and/or increased T helper-17 (Th17) cells play a central role in the pathogenesis of PE. ${ }^{2627}$ Thus, we investigated whether the adverse effect observed with FMT was associated with changes in Treg/Th17 proportions. Treg and Th17 cells were defined as coexpressing $\mathrm{CD} 4{ }^{+} \mathrm{CD} 25^{+}$Foxp $^{+}$ and $\mathrm{CD} 4^{+} \mathrm{IL}-17 \mathrm{~A}^{+}$, respectively. The number of Treg and Th17 cells was expressed as a percentage of CD4 $+\mathrm{T}$ cells for each subject and compared among different groups. Flow cytometric analysis of splenocytes and small intestinal lamina propria lymphocytes revealed a significant reduction in Treg cells and a marked increase in Th17 cells after FMT from patients with PE for 62 days compared with those in the NP-FMT and control groups (figure 3A-D). Moreover, the Treg/Th17 cell ratios were lower in PE-FMT mice than in NP-FMT and control mice (figure 3E). We further examined inflammatory gene expression in the ileum to detect alterations in immunological responses. As shown in figure 3F, PE-FMT mice exhibited significantly higher $I l 1 \beta, C c l 3$, Cxcl1 and Vegf levels than NP-FMT or control mice. Collectively, these data indicate that the faecal microbiota from patients with PE disrupted the mucosal and systematic immunological response.

\section{FMT in patients with PE microbiota impairs intestinal barrier function}

Impaired immunological responses in the intestine are known to be associated with gut barrier dysfunction. ${ }^{28}$ We investigated if faecal bacteria from patients with PE affected gut barrier function. Intestinal permeability was monitored by faecal albumin content, which was significantly increased in the PE-FMT group 

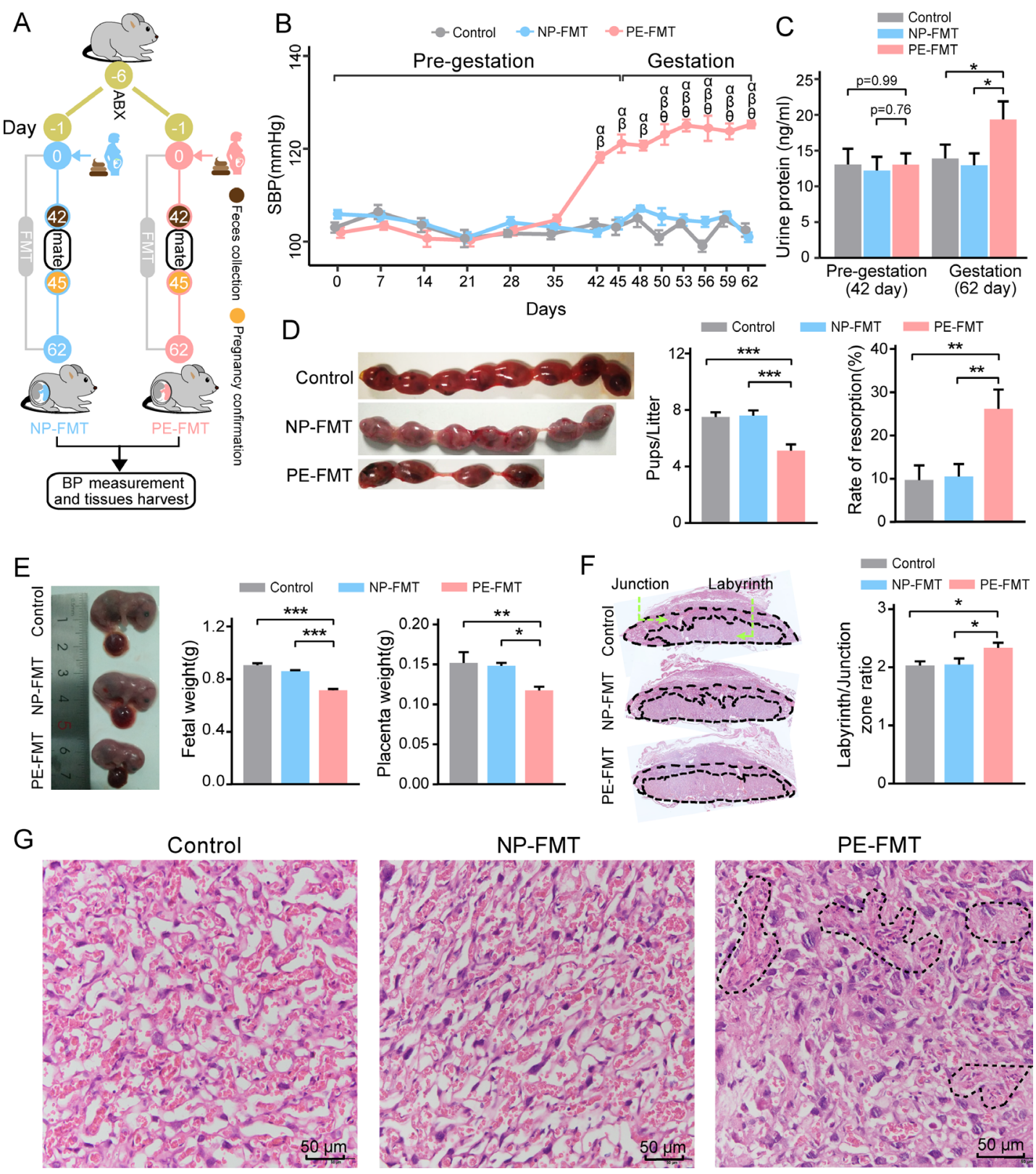

Figure 2 Administration of faecal bacteria from patients with PE to mice induced a series of PE-like phenotypes. (A) Schematic representation of FMT. Fresh faecal from three PE and three NP donors were mixed and used as a single source for PE-FMT and NP-FMT mice, respectively. Following antibiotic treatment, the recipient mice were randomly divided and orally inoculated daily for three consecutive days and two times per week for 59 days with prepared faecal contents. (B) SBP of the three groups was evaluated pregestation and postgestation ( $n=8$ for control and PE-FMT, $n=10$ for NP-FMT). $\alpha$ indicates $p<0.01$ versus NP-FMT group on the corresponding day; $\beta$ indicates $p<0.01$ versus control group on the corresponding day. $\theta$ indicates $p<0.05$ for each corresponding gestational day versus the pregestational day (42 days post-FMT) by two-tailed Student's t-test. (C) Urine protein concentration was measured at 42 days (prior to mating) and 62 days (17 days of gestation) post-FMT ( $\mathrm{n}=8$ for control and PE-FMT, $n=10$ for NP-FMT). (D) Number of pups and resorption rate per litter ( $n=8$ for control and PE-FMT, $n=10$ for NP-FMT). (E) Placental weight and fetal weight significantly decreased in PE-FMT $(n=41)$ compared with in NP-FMT $(n=76)$ and controls $(n=59)$ at 17 days of gestation. (F) Representative images of H\&E-stained midsagittal placental tissue sections used in histomorphological analysis. Zones are marked and indicated by labyrinth zone and junctional zone. The ratio between the labyrinth/junctional zones was enhanced in PE-FMT $(n=14)$ compared with in NP-FMT ( $n=16)$ and controls ( $n=16)$. (G) Rpresentative H\&E staining of the labyrinth zone of recipient mouse placentas. Typical lesions are marked with dotted line (original magnification, $\times 400$; scale bar $=50 \mu \mathrm{m}$ ). Data are presented as the mean $\pm S E M(B-F)$. ${ }^{*} P<0.05,{ }^{* *} P<0.01,{ }^{* *} P<0.001$ by one-way analysis of variance following Duncan's multiple range test. $A B X$, antibiotics; BP, blood pressure; FMT, faecal microbiota transplantation; NP, normotensive pregnant; PE, pre-eclampsia; SBP, systolic blood pressure.

(figure 4A). FMT markedly increased plasma endotoxin levels in PE-FMT mice (figure 4B). In addition, faecal microbiota from patients with PE decreased Claudin4 and Occludin gene expression in the colon compared with that in NP-FMT or control mice (figure 4C). Similarly, barrier-forming tight-junction protein
ZO-1 and ZO-2, claudin-4 and occludin protein levels in the colon were markedly lower according to immunohistochemistry and western blot analyses (figure 4D-H). We next examined the effect of FMT on intestinal histopathological changes. There was no obvious difference in the morphology of the epithelium in 

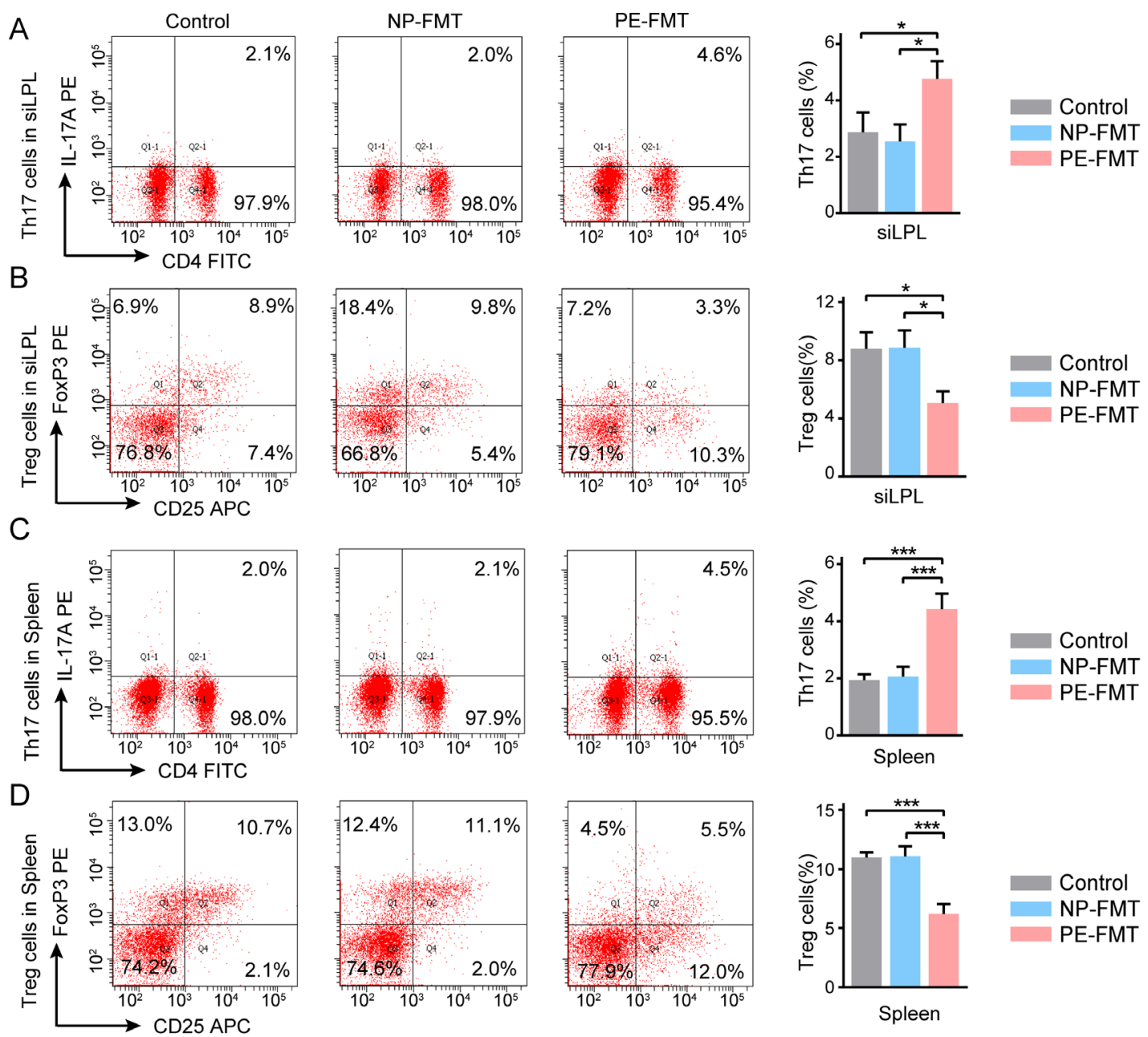

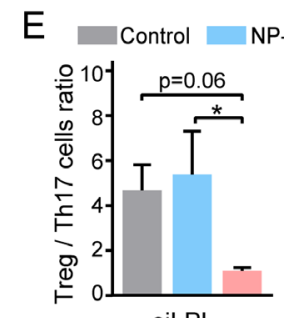

siLPL
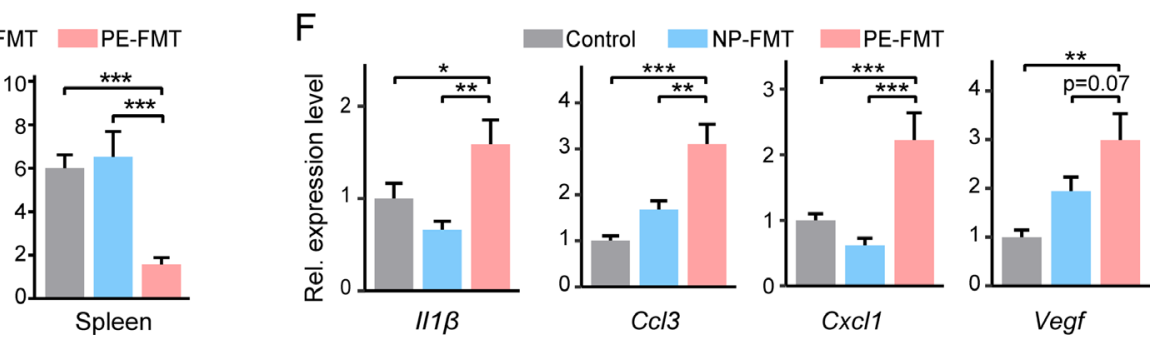

Figure 3 FMT induced immunological imbalance. Treg and Th17 cells were analysed for coexpression of CD4 $4^{+} \mathrm{CD} 25^{+}$Foxp3 ${ }^{+}$and $\mathrm{CD} 4^{+}$IL- $17 \mathrm{~A}^{+}$by flow cytometry, respectively. The number of Treg and Th17 cells was expressed as a percentage of CD4 ${ }^{+} \mathrm{T}$ cells for each subject and compared among different groups. (A-D) Percentages of Th17 cells in siLPL and spleen, and Treg cells in siLPL and spleen were determined ( $n=8$ mice in each group). (E) Treg/Th17 cell ratios in siLPL and spleen of the three groups are shown ( $n=8$ mice in each group). (F) Messenger RNA levels of key cytokines and chemokines in the ileum $(n=7-8)$. Data are presented as the mean \pm SEM $(A-F) ;{ }^{*} P<0.05,{ }^{*} P<0.01,{ }^{*} * P<0.001$ by one way analysis of variance following Duncan's multiple range test. FMT, faecal microbiota transplantation; NP, normotensive pregnant; PE, pre-eclampsia; siLPL, small intestinal lamina propria lymphocyte; Th17, helper $17 \mathrm{~T}$; Treg, regulatory T.

the ileum and colon among the three groups, except for mild infiltration of inflammatory cells in the colon of PE-FMT mice (online supplementary figure S7). Furthermore, significant correlations between the abundance of the gut microbiome, gut barrier and immune index were observed (figure 4I). These data demonstrate that the intestinal barrier was disrupted by faecal bacteria from patients with PE.

\section{Bacterial translocation is associated with inflammation in the placenta}

Increased gut permeability can lead to gut bacteria or bacterial component translocation into the extraintestinal tissues. ${ }^{29} 30 \mathrm{We}$ next detected bacteria in human and mouse placentas by quantitative PCR to evaluate the bacterial 16S rRNA gene. The levels of total bacterial DNA were obviously increased in the PE and PE-FMT placentas (figure 5A,C). To confirm these results, we performed ISH using the probe EUB338 targeting bacterial 16S rRNA. Probe specificity was also tested using sections treated with a sequence scrambled probe. More bacterial signals were visualised in the villous tissue of PE placenta and junction layer of PE-FMT placenta. No staining for scrambled probe controls was observed but was detected in the EUB338-probed sections (figure 5B,D and online supplementary figures S8 and S9). As shown in online supplementary figure S10A,B, the detection rate 
A
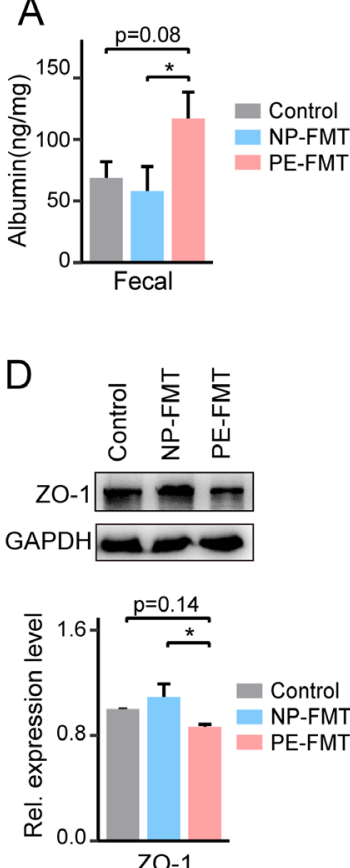

$\mathrm{H}$
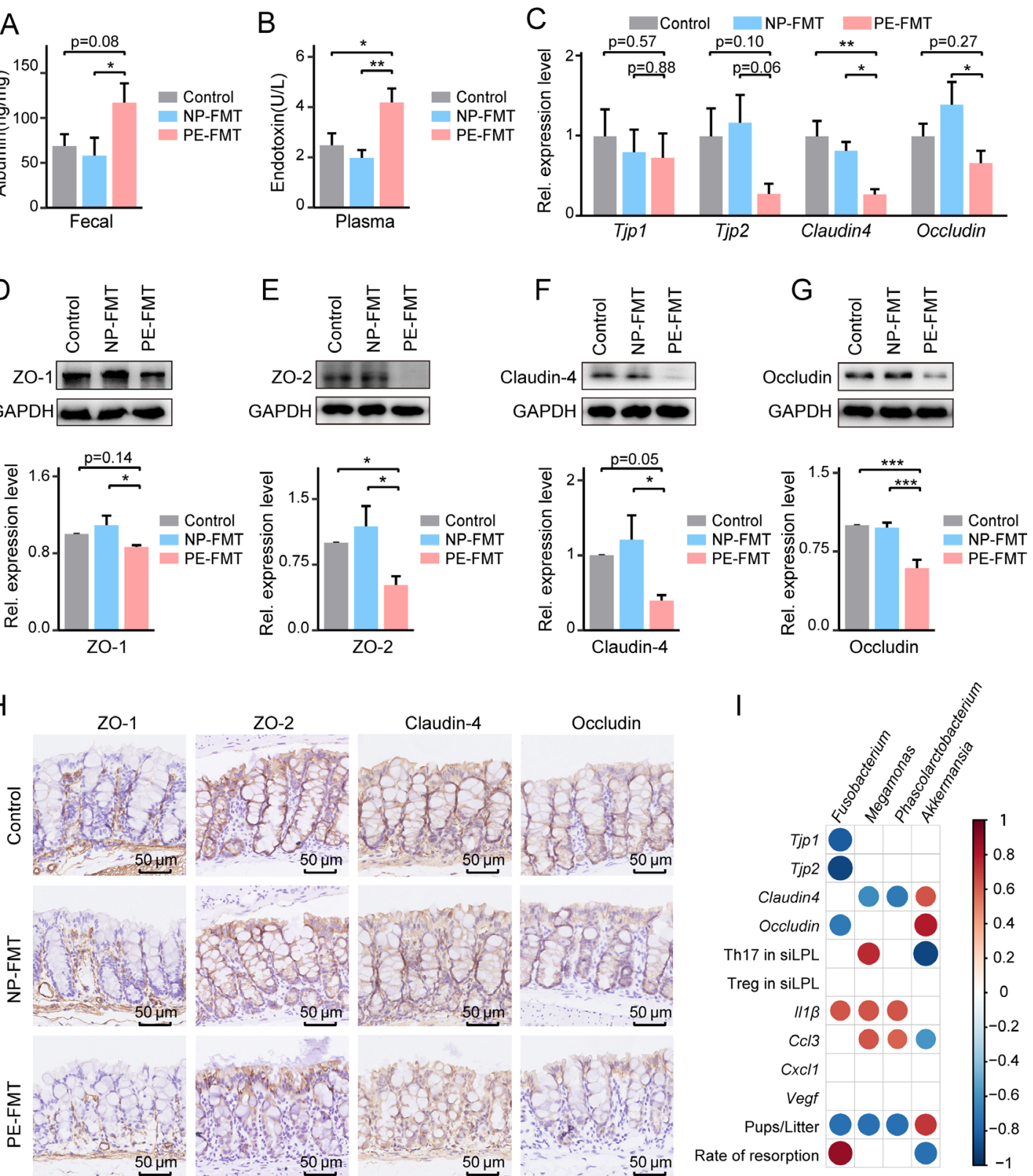

Figure 4 FMT impaired gut barrier function. (A) Faecal albumin content ( $n=8$ in each group). (B) Endotoxin level in plasma ( $n=8$ in each group). (C) Tight junction messenger RNA levels in the colon ( $n=6-8$ in each group). (D-G) Tight junction protein ZO-1, ZO-2, occludin and claudin-4 protein levels in the colon ( $n=4$ in each group). (H) Representative image of ZO-1, ZO-2, occludin and claudin- 4 immunofluorescence staining in colon tissues (original magnification, $\times 400$; scale bar $=50 \mu \mathrm{m}$ ). (I) Correlations between the relative abundance of selected bacterial genera, genes and phenotypes. Correlations with $p<0.05$ are shown. Spearman's rho statistic adjusted by the Benjamini and Hochberg method. Data are presented as the mean \pm SEM $(A-G)$. ${ }^{*} P<0.05,{ }^{* *} P<0.01,{ }^{* *} P<0.001$ by one-way analysis of variance following Duncan's multiple range test. FMT, faecal microbiota transplantation; NP, normotensive pregnant; PE, pre-eclampsia; siLPL, small intestinal lamina propria lymphocyte; ZO, zonula occluden.

of Fusobacterium DNA was higher in PE placental samples than in NP samples according to quantitative PCR and ISH probing for the Fusobacterium $16 \mathrm{~S}$ rRNA gene.

During experimental processing, we monitored contamination by adding DNA-free water during extraction and amplification (DNA extraction from blank controls and no-template amplification controls), as well as a sterile swab during placental sampling (sampling blank controls). As shown in figure 5E, placental metagenomes from human and mouse samples were significantly distinct in their clustering from negative controls. To exclude the possibility that the placental microbiota occurrence was due to contamination by bacteria in the environment, $16 \mathrm{~S}$ metagenomics decontamination analysis was rigorously performed using the $\mathrm{R}$ package decontam and SourceTracker (online supplementary figure S8C,D). According to the no-contaminant filtered data, $\mathrm{PCoA}$ revealed the significant separation of two clusters of PE and normotension groups and NP-FMT and PE-FMT groups (figure 5F,G). LEfSe analysis identified different taxa between the PE and NP groups, showing that Pseudomonas and Enterobacteriaceae were enriched, whereas Ruminococcaceae and Flavobacteriia were depleted in patients with PE (online supplementary figure S10E,F). These data suggest that a low but consistently detectable biomass of bacteria DNA present in the placenta is taxonomically distinct from potential environmental negative controls (online supplementary figure S10G). 

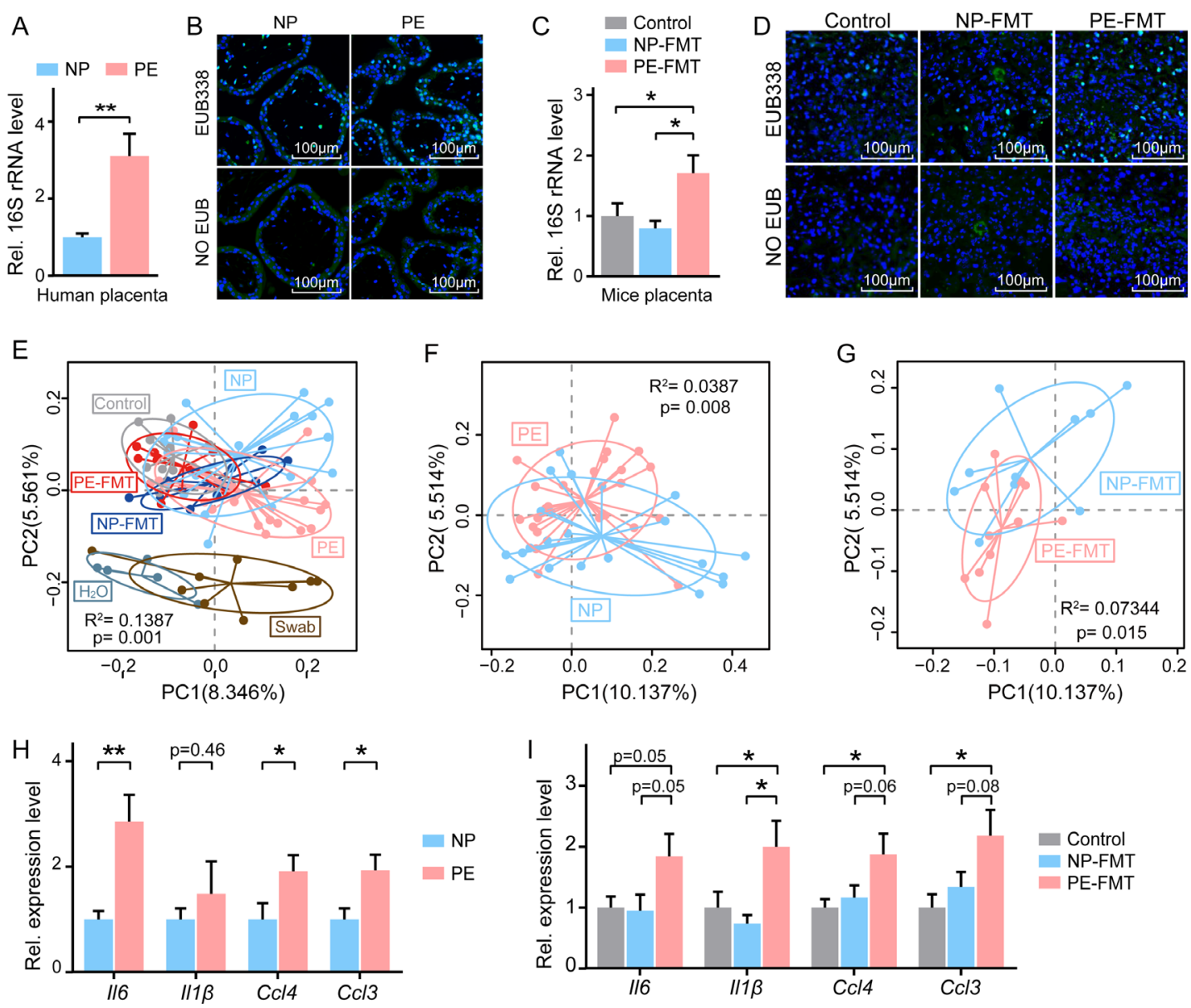

Figure 5 Bacterial translocation and inflammation in the placenta. (A) Total bacterial load indicated by the relative expression of 16S rRNA in normotension (NP) and PE placentas ( $n=20-22)$. (B) Representative in situ detection of bacteria in the NP and PE placentas. Placental tissue sections were stained with DAPI (blue) and probed with universal eubacterial probe EUB338 (green) and scrambled probe NOEUB. Original magnification: $\times 200$; scale bar $=100 \mu \mathrm{m}$. (C) Comparison of total bacterial load in the placenta of PE-FMT, NP-FMT and control mice ( $\mathrm{n}=8$ in each group). (D) Representative images of in situ staining of the junction zone of the recipient mice placentas. 165 probe EUB338 staining is shown in green, with nuclear stain (DAPI) in blue. Original magnification: $\times 200$; scale bar $=100 \mu \mathrm{m}$. (E) PCoA of unweighted UniFrac distances for all samples, coloured by sample type. The eigenvalues of axe PC1 and PC2 were $1.61(8.35 \%)$ and $1.07(5.56 \%)$, respectively. $(F, G)$ PCoA based on unweighted UniFrac distances comparing bacterial community structure of placenta samples between patients with PE and NP, and PE-FMT and NP-FMT mice. The eigenvalues of axe PC1 and PC2 were $1.63(10.14 \%)$ and $0.89(5.51 \%)$, respectively (F). The eigenvalues of axe PC1 and PC2 were $1.63(10.14 \%)$ and $0.89(5.51 \%)$, respectively $(\mathrm{G})$. (H) mRNA levels of key cytokines and chemokines in PE and NP placentas $(n=21-24)$. (I) mRNA levels of key cytokines and chemokines in mouse placentas $(n=7-8)$. Differences in beta diversity were tested by permutational multivariate ANOVA (Adonis) ( $E-G)$. Data are presented as the mean $\pm \mathrm{SEM}(\mathrm{A}, \mathrm{C}, \mathrm{H}, \mathrm{I}) .{ }^{*} \mathrm{P}<0.05,{ }^{*} \mathrm{P}<0.01$; two-tailed Student's $t$ test $(\mathrm{A}, \mathrm{H})$; one-way ANOVA following Duncan's multiple range test $(C, I)$. ANOVA, analysis of variance; FMT, faecal microbiota transplantation; mRNA, messenger RNA; NP, normotensive pregnant; PCoA, principal coordinate analysis; PE, pre-eclampsia; rRNA, ribosomal RNA.

Proinflammatory cytokine and chemokines are thought to play key roles in the pathophysiology of PE. ${ }^{3132}$ We next examined alterations in chemokine and proinflammatory cytokine expression in the placenta. Particularly, the levels of IL6, Il1 $\beta$, $\mathrm{Ccl} 3$ or $\mathrm{Ccl} 4$ messenger RNA were significantly increased in the $\mathrm{PE}$ group compared with those in the NP group for both human and mice placentas (figure $5 \mathrm{H}, \mathrm{I}$ ).

\section{DISCUSSION}

$\mathrm{PE}$ is one of the most severe diseases during pregnancy that not only leads to pregnancy abortion but also is life threatening. ${ }^{1}$ Effective methods for the prediction, prevention and treatment of this disease are lacking because its aetiology remains unclear. ${ }^{3}$ The present study aimed to seek these answers from the gut. We found that the gut microbiome was dramatically shifted in patients with PE. Thus, using FMT, we proved that the gut dysbiosis can cause pre-eclamptic systems. One possible mechanism through which this happens might be gut barrier destruction and the translocation of pathogenic bacteria from the gut to the placenta, causing abnormal immune responses, as we identified higher levels of total bacteria and Fusobacterium in the placenta of both human and mice with PE. Our study proposed that a 'gut-placenta' axis could play a crucial role in understanding the aetiology of PE.

The gut dysbiosis in PE observed in our study was obvious and echoes the findings of previous reports. Because the human gut microbiome is highly heterogeneous among individuals, microbiomes in healthy and diseased individuals are usually overlapped with partial differentiation when analysed with unsupervised learning. ${ }^{8}$ Nevertheless, in this study, the gut microbiome in PE clearly deviated from that in the controls and showed very little overlap, indicating severe dysbiosis in PE. More interesting 
were the dysbiosis patterns, such that Faecalibacterium and Akkermansia were depleted, whereas Fusobacterium and Veillonella were enriched in PE. Similar to the microbiota profile of non-pregnant hypertensive patients, ${ }^{11}$ Faecalibacterium and Akkermansia, which are involved in producing intestinal epithelial nutrition, maintaining immune homeostasis and strengthening intestinal barrier functions, were depleted in patients with PE. ${ }^{33}$ Fusobacterium and Veillonella are mainly opportunistic pathogens and are associated with chronic inflammatory conditions and adverse pregnancy outcomes. ${ }^{35-37}$ Moreover, Liu et al found that these bacteria were changed in the intestine of patients with PE in America and South China, indicating that the dysbiosis patterns for PE are consistent across populations and regions. ${ }^{14} 1538$ Thus, the dysbiosis pattern in PE reported by our research is reliable.

Fusobacterium could be the key regulator of the gutplacenta axis during PE. The enrichment of Fusobacterium in $\mathrm{PE}$ is especially worth noting because it is a newly identified key pathogen involved in promoting colorectal cancer. ${ }^{39}$ The mechanism involved is that Fusobacterium can affect apoptosis, cellular proliferation and DNA repair through its attachment to epithelial cells via Fusobacterium adhesion A and its activation of the immune response via the nuclear factor- $\mathrm{\kappa B}$ signalling pathways. ${ }^{40}$ In other words, Fusobacterium is especially good at disrupting intestinal epithelia leading to gut leakage. ${ }^{41}$ A possible consequence of a leaky gut is bacterial translocation, as we have identified more total bacteria and Fusobacterium in the placenta of both women and mice with PE. Moreover, Amarasekara et al also found the increase of Fusobacterium in PE placentas, which may invade endothelial cells and elicit local inflammation. ${ }^{42} 43$ The existence of a placenta microbiome is at the centre of debate ${ }^{4445}$; nevertheless, our standpoint is that bacteria can translocate into placenta in pathological conditions, which is supported by recent studies. ${ }^{46}$ Moreover, we carefully maintained sterile conditions during placenta sampling and used controls to monitor contaminations. Even though the possibility of contamination cannot be theoretically ruled out currently, we believe that future studies focusing on Fusobacterium can help gain insight into the gut-placenta axis during PE.

Excessive maternal inflammation, commonly involved in PE pathogenesis by an imbalance of Treg/Th17, is also a putative result of gut dysbiosis. ${ }^{26}{ }^{47}$ In the current study, PE-FMT mice exhibited a mucosal and systematic imbalance of Treg and Th17 cells and exaggerated inflammation in the placenta. This increased the inflammatory cytokines, leading to placental oxidative stress and vascular dysfunction, resulting in rejection of the fetus and the development of hypertension..$^{32}$ We found that gut dysbiosis in PE can elevate the host's BP, which is a major manifestation of PE. It has been reported that the gut microbiome is altered on entry into pregnancy ${ }^{48}$; therefore, we deduce that in some cases, a dysbiosis status in pregnancy can elevate the BP, leading to PE. This deduction provides a novel view of PE aetiology, which needs further research. Interestingly, mice receiving faecal material from patients with PE showed elevated BP even before pregnancy, indicating that the hypertensive effect of gut dysbiosis is independent of pregnancy. It is also unclear why some women have this PE-prone dysbiosis during pregnancy; for example, whether the dysbiosis occurs due to hormone changes or diet shifting needs to be investigated in the future.

The present study has several limitations. First, for the mouse FMT experiment, we used whole faecal materials that included bacteria, fungi, virus, phages, proteins and metabolites from both bacteria and the host. Therefore, it is not precisely clear which component from the faeces functions in PE. It is, thus, important to use multiomics techniques, inactivated faeces and isolated bacteria/metabolites to affirm the key factors contributing to PE. Second, even though we have used three methods, including sequencing, ISH and quantitative PCR, and identified bacteria in the placenta, these methods do not indicate the liveness of the detected bacteria. Future studies that isolate live bacteria from placenta can certainly add credibility to our findings. Lastly, even though we have detected the enrichment of Fusobacterium in the gut and placenta and hypothesised its translocation from the gut to the placenta, the exact route through which Fusobacterium completes its translocation also needs further investigation.

Overall, we identified here obvious gut dysbiosis in patients with PE and proved that this dysbiosis can cause pre-eclamptic systems. The possible underlying mechanisms is that dysbiosis influences the host's BP and that gut Fusobacterium translocates into the placenta, causing local placental inflammation. Cohort studies that follow up women from early pregnancy to observe their microbiome dynamics, BP changes and PE occurrence will offer critical evidence linking the gut microbiome to PE. In conclusion, our study indicates the role of the gut-placenta axis in PE, providing new insights into PE aetiology.

\section{Author affiliations}

${ }^{1}$ Department of Obstetrics and Gynecology, Southern Medical University Nanfang Hospital, Guangzhou, Guangdong, China

${ }^{2}$ Microbiome Medicine Center, Division of Laboratory Medicine, Zhujiang Hospital, Southern Medical University, Guangzhou, Guangdong, China

${ }^{3}$ Microbiome Research Centre, St George and Sutherland Clinical School, University of New South Wales, Sydney, New South Wales, Australia

${ }^{4}$ Department of Obstetrics and Gynecology, First Affiliated Hospital of Nanchang University, Nanchang, Jiangxi, China

${ }^{5}$ Department of Internal Medicine, Affiliated Hospital, Chengdu University, Chengdu, Sichuan, China

Contributors $Y Y, H Z, L H, Y H$ and $X C$ designed the study and prepared the manuscript. $X C, M L, X Y, Y F, L Z, Y C, Y H, G K, M Z$ and $Z W$ collected the samples and conducted the experiments. PL, HZ, YH, XC, WT and $\mathrm{HH}$ analysed the data. All authors approved the final version of the manuscript.

Funding This investigation was financially supported by the National Natural Science Foundation of China (81925026, 81703078, 81771609, 81800746 and 31570497), the National Key Research and Development Program of China (2019YFA0802300), the Natural Science Foundation of Guangdong Province (2017A010105025) and China Postdoctoral Science Foundation (2018M633077).

Competing interests None declared.

Patient consent for publication Not required.

Ethics approval Nanfang Hospital.

Provenance and peer review Not commissioned; externally peer reviewed.

Data availability statement Data are available in a public, open access repository.

Open access This is an open access article distributed in accordance with the Creative Commons Attribution Non Commercial (CC BY-NC 4.0) license, which permits others to distribute, remix, adapt, build upon this work non-commercially, and license their derivative works on different terms, provided the original work is properly cited, appropriate credit is given, any changes made indicated, and the use is non-commercial. See: http://creativecommons.org/licenses/by-nc/4.0/.

\section{ORCID iD}

Xia Chen http://orcid.org/0000-0002-7915-4568

\section{REFERENCES}

1 Mol BWJ, Roberts CT, Thangaratinam S, et al. Pre-eclampsia. Lancet 2016;387:999-1011.

2 Anon. ACOG practice Bulletin No. 202: gestational hypertension and preeclampsia. Obstet Gynecol 2019;133:e1-25.

3 Brown MA, Magee LA, Kenny LC, et al. Hypertensive disorders of pregnancy: ISSHP classification, diagnosis, and management recommendations for international practice. Hypertension 2018;72:24-43. 
4 Udenze IC. Association of pre-eclampsia with metabolic syndrome and increased risk of cardiovascular disease in women: a systemic review. Niger J Clin Pract 2016;19:431-5.

5 Jabalie G, Ahmadi M, Koushaeian L, et al. Metabolic syndrome mediates proinflammatory responses of inflammatory cells in preeclampsia. Am J Reprod Immunol 2019;81:e13086.

6 Rodie Vet al. Pre-Eclampsia and cardiovascular disease: metabolic syndrome of pregnancy? Atherosclerosis 2004;175:189-202.

7 Hooijschuur MCE, Ghossein-Doha C, Kroon AA, et al. Metabolic syndrome and preeclampsia. Ultrasound Obstet Gynecol 2019;54:64-71.

$8 \mathrm{He} \mathrm{Y,} \mathrm{Wu} \mathrm{W,} \mathrm{Zheng} \mathrm{H-M,} \mathrm{et} \mathrm{al.} \mathrm{Regional} \mathrm{variation} \mathrm{limits} \mathrm{applications} \mathrm{of} \mathrm{healthy} \mathrm{gut}$ microbiome reference ranges and disease models. Nat Med 2018;24:1532-5.

9 Wang J, Zheng J, Shi W, et al. Dysbiosis of maternal and neonatal microbiota associated with gestational diabetes mellitus. Gut 2018;67:1614-25.

10 Zhao L. The gut microbiota and obesity: from correlation to causality. Nat Rev Microbiol 2013;11:639-47.

11 Li J, Zhao F, Wang Y, et al. Gut microbiota dysbiosis contributes to the development of hypertension. Microbiome 2017;5.

12 Kim S, Goel R, Kumar A, et al. Imbalance of gut microbiome and intestinal epithelia barrier dysfunction in patients with high blood pressure. Clin Sci 2018;132:701-18.

13 Kanbay M, Onal EM, Afsar B, et al. The crosstalk of gut microbiota and chronic kidney disease: role of inflammation, proteinuria, hypertension, and diabetes mellitus. Int Urol Nephrol 2018;50:1453-66.

14 Liu J, Yang H, Yin Z, et al. Remodeling of the gut microbiota and structural shifts in preeclampsia patients in South China. Eur J Clin Microbiol Infect Dis 2017;36:713-9.

15 Lv L-J, Li S-H, Li S-C, et al. Early-Onset preeclampsia is associated with gut microbial alterations in antepartum and postpartum women. Front Cell Infect Microbiol 2019:9:224.

16 Gong S, Yan Z, Liu Z, et al. Intestinal microbiota mediates the susceptibility to polymicrobial Sepsis-Induced liver injury by granisetron generation in mice. Hepatology 2019;69:1751-67.

17 Chen X, Xiu M, Xing J, et al. Lanthanum chloride inhibits LPS mediated expressions of pro-inflammatory cytokines and adhesion molecules in HUVECs: involvement of NF-KB-Jmjd3 signaling. Cell Physiol Biochem 2017;42:1713-24.

18 Choi YS, Kim YC, Baek KJ, et al. <em $>$ In Situ</em > Detection of Bacteria within Paraffin-embedded Tissues Using a Digoxin-labeled DNA Probe Targeting 16S rRNA. JoVE 2015:e52836.

19 Daims H, Brühl A, Amann R, et al. The domain-specific probe EUB338 is insufficient for the detection of all bacteria: development and evaluation of a more comprehensive probe set. Syst App/ Microbiol 1999;22:434-44.

20 Edgar RC. Search and clustering orders of magnitude faster than blast. Bioinformatics 2010;26:2460-1.

21 McDonald D, Price MN, Goodrich J, et al. An improved Greengenes taxonomy with explicit ranks for ecological and evolutionary analyses of bacteria and archaea. Isme J 2012;6:610-8.

22 Segata N, Izard J, Waldron L, et al. Metagenomic biomarker discovery and explanation. Genome Biol 2011;12:R60.

23 Davis NM, Proctor DM, Holmes SP, et al. Simple statistical identification and removal of contaminant sequences in marker-gene and metagenomics data. Microbiome 2018;6.

24 Knights D, Kuczynski J, Charlson ES, et al. Bayesian community-wide cultureindependent microbial source tracking. Nat Methods 2011;8:761-3.10.1038/ nmeth. 1650

25 Benjamini Y, Yekutieli D. The control of the false discovery rate in multiple testing under dependency. Ann Statist 2001;29:1165-88.

26 Eghbal-Fard S, Yousefi M, Heydarlou H, et al. The imbalance of Th17/Treg axis involved in the pathogenesis of preeclampsia. J Cell Physiol 2019;234:5106-16.
27 Zhang Y, Liu Z, Tian M, et al. The altered PD-1/PD-L1 pathway delivers the 'one-two punch' effects to promote the Treg/Th17 imbalance in pre-eclampsia. Cell Mol Immunol 2018;15:710-23.

28 Su L, Nalle SC, Shen L, et al. Tnfr2 activates MLCK-dependent tight junction dysregulation to cause apoptosis-mediated barrier loss and experimental colitis. Gastroenterology 2013;145:407-15.

29 Wu L-L, Peng W-H, Kuo W-T, et al. Commensal bacterial endocytosis in epithelial cells is dependent on myosin light chain kinase-activated brush border fanning by interferon- $\gamma$. Am J Pathol 2014;184:2260-74.

30 Manfredo Vieira S, Hiltensperger M, Kumar V, et al. Translocation of a gut pathobiont drives autoimmunity in mice and humans. Science 2018;359:1156-61.

31 Raghupathy R. Cytokines as key players in the pathophysiology of preeclampsia. Med Princ Pract 2013:22:8-19.

32 Holder BS, Tower CL, Jones CJP, et al. Heightened pro-inflammatory effect of preeclamptic placental microvesicles on peripheral blood immune cells in Humans1. Biol Reprod 2012;86:103.

33 Hiippala K, Jouhten H, Ronkainen A, et al. The potential of gut Commensals in reinforcing intestinal barrier function and alleviating inflammation. Nutrients 2018;10:988.

34 Routy B, Gopalakrishnan V, Daillère R, et al. The gut microbiota influences anticancer immunosurveillance and general health. Nat Rev Clin Oncol 2018;15:382-96.

35 Kummen M, Holm K, Anmarkrud JA, et al. The gut microbial profile in patients with primary sclerosing cholangitis is distinct from patients with ulcerative colitis without biliary disease and healthy controls. Gut 2017;66:611-9.

36 De Cruz P, Kang S, Wagner J, et al. Association between specific mucosa-associated microbiota in Crohn's disease at the time of resection and subsequent disease recurrence: a pilot study. J Gastroenterol Hepatol 2015;30:268-78.

37 Barak S, Oettinger-Barak O, Machtei EE, et al. Evidence of periopathogenic microorganisms in placentas of women with preeclampsia. J Periodontol 2007;78:670-6.

38 DiGiulio DB, Callahan BJ, McMurdie PJ, et al. Temporal and spatial variation of the human microbiota during pregnancy. Proc Natl Acad Sci U S A 2015:112:11060-5.

39 Yu T, Guo F, Yu Y, et al. Fusobacterium nucleatum promotes chemoresistance to colorectal cancer by modulating autophagy. Cell 2017;170:e16:548-63.

40 Rubinstein MR, Wang X, Liu W, et al. Fusobacterium nucleatum promotes colorectal carcinogenesis by modulating $\mathrm{E}$-Cadherin/ $\beta$-Catenin signaling via its FadA adhesin. Cell Host Microbe 2013:14:195-206.

41 Han YW. Fusobacterium nucleatum: a commensal-turned pathogen. Curr Opin Microbiol 2015:23:141-7.

42 Amarasekara R, Jayasekara RW, Senanayake $\mathrm{H}$, et al. Microbiome of the placenta in pre-eclampsia supports the role of bacteria in the multifactorial cause of preeclampsia. J Obstet Gynaecol Res 2015;41:662-9.

43 Rustveld LO, Kelsey SF, Sharma R. Association between maternal infections and preeclampsia: a systematic review of epidemiologic studies. Matern Child Health $J$ 2008; 12:223-42

44 Seferovic MD, Pace RM, Carroll M, et al. Visualization of microbes by $16 \mathrm{~S}$ in situ hybridization in term and preterm placentas without intraamniotic infection. Am J Obstet Gynecol 2019:221:146.e1-146.e23.

45 de Goffau MC, Lager S, Salter SJ, et al. Recognizing the reagent microbiome. Nat Microbiol 2018:3:851-3.

46 de Goffau MC, Lager S, Sovio U, et al. Human placenta has no microbiome but can contain potential pathogens. Nature 2019;572:329-34.

47 Britton GJ, Contijoch EJ, Mogno I, et al. Microbiotas from humans with inflammatory bowel disease alter the balance of gut Th17 and RORyt+ regulatory T cells and exacerbate colitis in mice. Immunity 2019:50:212-24.

48 Koren 0, Goodrich JK, Cullender TC, et al. Host remodeling of the gut microbiome and metabolic changes during pregnancy. Cell 2012;150:470-80. 